Revista Latinoamericana de la Papa 24 (1): 47 - 57, 2020

Recebido: 04/04/2020 |Aceito: 14/05/2020

Publicado on-line: junho 2020

DOI: 10.37066/ralap.v24i1.389

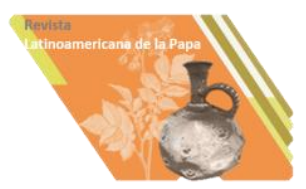

\title{
Seleção de clones canadenses de batata para caracteres agronômicos e de qualidade de fritura, no Sul do Brasil
}

\author{
Giovani Olegario da Silva ${ }^{1}$; Arione da Silva Pereira ${ }^{2 / *}$; Fernanda Quintanilha \\ Azevedo $^{3}$; Agnaldo Ferreira de Carvalho ${ }^{4}$; Caroline Marques Castro5; Elcio Hirano ${ }^{6}$
}

\section{Resumo}

Existe uma forte demanda da cadeia brasileira de batata por cultivares para o processamento na forma de palitos pré-fritos congelados. O objetivo deste trabalho foi avaliar clones de batata de origem canadense, quanto à adaptação ao Sul do Brasil. Foi avaliado um conjunto de 11 clones pertencentes ao "Centre de Recherche Les Buissons", QB, Canadá, e as cultivares Asterix e Markies (testemunhas). Experimentos foram realizados em Pelotas, RS, e Canoinhas, SC, Brasil, na primavera de 2019. O delineamento experimental foi blocos casualizados com quatro repetições e parcelas compostas por duas linhas de 10 plantas cada. Foram avaliados caracteres componentes de rendimento e de qualidade de fritura, vigor de planta e ciclo de desenvolvimento vegetativo. Além disso, em Pelotas, foi acessada a incidência de requeima (Phytophthora infestans). Os dados foram submetidos à análise de variância, de agrupamento de médias e de correlação. Foram observados clones com potencial produtivo, qualidade de fritura, vigor de planta e ciclo vegetativo similares às cultivares testemunhas. O clone BGB 476 destacou-se para caracteres agronômicos e caracteres de qualidade de fritura.

Palavras-chave adicionais: Solanum tuberosum L., rendimento, cor de fritura, peso específico, precocidade.

\footnotetext{
* Autor para correspondência. Correo electrónico: arione.pereira@embrapa.br

${ }^{1}$ Giovani Olegario da Silva. Embrapa, Canoinhas, Brasil.

${ }^{2}$ Arione da Silva Pereira. Embrapa, Pelotas, Brasil.

${ }^{3}$ Fernanda Quintanilha Azevedo. Embrapa, Pelotas, Brasil.

${ }^{4}$ Agnaldo Ferreira de Carvalho. Embrapa, Gama, Brasil.

${ }^{5}$ Caroline Marques Castro. Embrapa, Pelotas, Brasil.

${ }^{6}$ Elcio Hirano. Embrapa, Pelotas, Brasil.
} 


\section{Selection of Canadian potato clones for agronomic and frying quality traits, in South of Brazil}

\section{Summary}

The strong demand in the Brazilian potato chain for processing frozen French fries varieties, lead us to evaluate the adaptation of Canadian potato clones to the south of Brazil. We evaluated eleven clones belonging to the Center de Recherche Les Buissons, QB, Canada, and the cultivars Asterix and Markies (controls). Experiments were carried out in Pelotas, RS, and Canoinhas, SC, Brazil, in the spring season of 2019. The experimental design was a completely randomized blocks with four replications of plots composed by two rows with 10 plants each. Agronomic and frying quality traits were evaluated. Component traits of tuber yield and of frying quality, plant vigor and maturity were evaluated. Besides, in Pelotas, late blight (Phytophthora infestans) incidence was assessed. Individual and jointly variance analysis of variance, grouping of means and correlation analysis were performed on the data. The clone BGB 476 stood out for high tuber yield, mainly in Canoinhas, as well for good fry quality. The BGB 476 clone stood out for agronomic traits and for frying quality traits.

Keywords: Solanum tuberosum L., yield, fry color, specific gravity, earliness.

\section{Introdução}

A demanda por produtos industrializados de batata é crescente no Brasil (Pereira \& Silva, 2019). Isso se deve às mudanças nos hábitos de vida dos consumidores, dispensando menos tempo na preparação dos alimentos, e aumentando as refeições fora de casa, que é respondida com incremento nas cadeias de restaurantes, que por sua vez demandam produtos semiprocessados de batata de alta qualidade (Silva et al., 2018; Freitas et al., 2006; Haase, 2008; Cabezas-Serrano et al., 2009). Em batata para o processamento industrial na forma de "chips" ou palitos, os caracteres mais importantes são aqueles que conferem qualidade de fritura e rendimento industrial, tais como alto peso específico, baixo teor de açúcares redutores e ausência de distúrbios fisiológicos (Souza et al., 2011; Silva et al., 2018).

O peso específico é um caráter importante, por ser diretamente relacionado com o teor de massa seca nos tubérculos (Schippers, 1976), o qual, quanto mais elevado, resulta em maior rendimento na industrialização, por menor absorção de gordura, e melhor textura do produto final (Smith, 1975; Fernandes et al., 2010). O baixo teor de açúcares redutores evita o escurecimento dos produtos processados, que compromete a aparência e o sabor do produto frito (Stark e Love, 2003).

No Brasil há uma grande carência de cultivares de batata, com características para a fabricação de palitos pré-fritos congelados, e adaptação aos diversos ecossistemas onde há cultivo de batata. Além disso, é reconhecida a expertise canadense em relação ao desenvolvimento de cultivares para processamento. No entanto, as condições de cultivo das regiões produtoras daquele país são diferentes em vários aspectos daquelas observadas no Sul do Brasil (Silva et al., 2012; Moreira et al., 2015; Silva et al., 2018). 
$\mathrm{Na}$ província de Quebec, origem dos clones do presente estudo, devido às temperaturas extremamente baixas em alguns meses do ano, o cultivo de batata é realizado na primavera-verão, quando o fotoperíodo médio fica entre 16 a 18 horas e as temperaturas entre 6 e $30^{\circ} \mathrm{C}$, enquanto no Sul do Brasil são feitas duas safras principais, na primavera e no outono, visando escapar de temperaturas muito elevadas no verão e de geadas no inverno, e sob fotoperíodo médio próximo de 12 horas e temperaturas entre 8 e $26^{\circ} \mathrm{C}$ nestes períodos (Silva et al., 2019).

Em resposta ao fotoperíodo menor no Brasil, as plantas apresentam redução do ciclo de desenvolvimento vegetativo, antecipação da tuberização e maior competição por fotoassimilados entre o crescimento vegetativo e o enchimento dos tubérculos. Além disso, com menor fotoperíodo ocorre menor produção de fotoassimilados e, consequentemente, menor produção de tubérculos (Pinto et al., 2010; Silva et al., 2012).

Dessa forma, o objetivo deste trabalho foi avaliar um conjunto de clones de batata de origem canadense, quanto a caracteres agronômicos e de qualidade de fritura, para adaptação ao Sul do Brasil.

\section{Material e Métodos}

Foi avaliado um conjunto de 11 clones oriundos do "Centre de Recherche Les Buissons" - CRLB, Baie-Comeau, QB, Canadá (49 $13^{\prime} \mathrm{N}, 68^{\circ} 9^{\prime} \mathrm{O}, 25$ manm), em comparação com Asterix e Markies, as duas cultivares mais utilizadas pela indústria brasileira no processamento de batata na forma de palitos pré-fritos congelados.

Os experimentos foram realizados no campo experimental da Estação Experimental de Canoinhas, Canoinhas,
SC ( $\left.26^{\circ} 10^{\prime} \mathrm{S}, 5^{\circ} 23^{\prime} \mathrm{O}, 839 \mathrm{manm}\right)$ e no campo experimental da sede da Embrapa Clima Temperado, Pelotas, RS (31 $41^{\prime} \mathrm{S}$, $\left.52^{\circ} 26^{\prime} \mathrm{O}, 60 \mathrm{manm}\right)$, Brasil, na safra de primavera de 2019. Em Pelotas, o plantio foi realizado no dia 21 de agosto de 2019, e em Canoinhas em 12 de agosto de 2019. $\mathrm{O}$ delineamento experimental foi blocos casualizados com quatro repetições de parcelas compostas por duas linhas de 10 plantas espaçadas em $0,35 \mathrm{~m}$ entre plantas e $0,75 \mathrm{~m}$ entre linhas, totalizando $5,25 \mathrm{~m}^{2}$.

A fertilização foi aplicada no sulco de plantio, usando 2,7 t.ha ${ }^{-1}$ da fórmula comercial NPK 05-20-10 em Pelotas, e 3,5 t.ha ${ }^{-1}$ da fórmula comercial NPK 0414-08 em Canoinhas. A amontoa foi realizada 30 dias após o plantio, e os demais tratos culturais e fitossanitários seguiram as recomendações para a cultura na região (Pereira, 2010).

Aos 70 dias após o plantio (DAP), período que coincide com a floração plena da maior parte dos clones, foi avaliado o vigor vegetativo das plantas (tamanho das plantas), atribuindo-se notas de 1 a 9 (1- baixo; 9- alto), de acordo com escala adaptada de Pereira et al. (2017). Aos 100 dias DAP, precedendo a colheita, foi avaliado o ciclo vegetativo das plantas, atribuindo-se notas de 1 a 9 (1- tardio; 9- precoce), segundo metodologia de Silva et al. (2012). Aos 80 DAP, foi realizada em Pelotas-RS, a determinação da porcentagem de plantas da parcela (incidência) acometidas pela doença requeima (Phytophthora infestans).

A colheita foi efetuada 100 DAP em ambos os locais. Os tubérculos de cada parcela foram classificados quanto ao diâmetro transversal em comerciais $(>45$ $\mathrm{mm}$ ) e não comerciais ( $\leq 45 \mathrm{~mm}$ ) (Lyra $e t$ $a l ., 2015$ ), contados e pesados, obtendo-se dados de massa total de tubérculos $(\mathrm{kg}$. 
parcela $\left.^{-1}\right)$, massa de tubérculos comerciais $\left(\mathrm{kg} . \mathrm{parcela}^{-1}\right)$. e massa média de tubérculos $(\mathrm{g})$. $\mathrm{O}$ peso específico foi medido em amostras de tubérculos de tamanho comercial, com utilização de hidrômetro da "Snack Food Association" (Arlington, VA, EUA) (Kumar et al., 2007). A cor de fritura foi avaliada, utilizando amostras de três tubérculos sadios de tamanho comercial por parcela. Para isso, cinco fatias de cerca de $2,0 \mathrm{~mm}$ de espessura foram cortadas de cada tubérculo e fritas em gordura vegetal à temperatura inicial de $180^{\circ} \mathrm{C}$ até cessar a borbulha. A cor de fritura foi avaliada atribuindo notas de 1 a 9 (1- escura, 9clara), de acordo com escala de TiemensHulscher et al. (2013). Os dados de massa de tubérculos foram convertidos em t.ha ${ }^{-1}$. Os dados de massa de tubérculos comercial e de peso específico foram transformados por $\sqrt{x+0,50}$. Posteriormente foram realizadas análises de variância individuais para cada local, no modelo em blocos ao acaso, e foram analisados conjuntamente no modelo fatorial (Cruz et al., 2014) seguido pela análise de agrupamento de médias por Scott-Knott ao nível de $5 \%$ de probabilidade. $\mathrm{O}$ coeficiente de variação experimental $(\mathrm{CVe})$ e o coeficiente de variação genética $(\mathrm{CVg})$ foram estimados, por meio das fórmulas $C V=100 \sqrt{Q M e / m e ́ d i a ~} \quad$ e $C V g=100 \sqrt{\sigma g / m e ́ d i a}$, respectivamente (Cruz, 2013). Também foram calculadas as correlações simples entre a incidência de requeima e os demais caracteres avaliados em PelotasRS; utilizando-se o aplicativo computacional Genes (Cruz, 2013).

\section{Resultados e Discussão}

As análises de variância conjunta revelaram diferenças significativas (p $<0,05)$ entre genótipos. A interação genótipo $\mathrm{x}$ ambiente foi significativa para todos os caracteres, exceto peso específico, indicando que os genótipos tiveram comportamento diferente em Pelotas e Canoinhas, ou baixa estabilidade frente aos ambientes (Tabela 1). Enquanto a análise de variância para a incidência de requeima em Pelotas revelou que não houve diferenças significativas entre os genótipos (dados não apresentados). Os valores do coeficiente de variação ambiental foram maiores para os caracteres componentes da produtividade comercial de tubérculos e para a porcentagem de plantas com requeima em Pelotas (Tabelas 1 e 2). Quanto aos caracteres de rendimento, que são quantitativos, é esperado maior coeficiente de variação ambiental (Silva et al., 2006). Entretanto, a relação entre o coeficiente de variação genético e o coeficiente de variação ambiental $(\mathrm{CVg} / \mathrm{CV})$ maior do que a unidade, sugerindo superioridade de variação de ordem genética, conferindo maior confiabilidade às estimativas (Cruz et al., 2014). 
Tabela 1. Resumo das análises de variância conjunta e individuais para caracteres de rendimento de tubérculos e de qualidade de fritura, de 11 clones avançados canadenses e duas cultivares de batata, na safra de primavera de 2019, em Canoinhas, SC, e Pelotas, RS, Brasil.

\begin{tabular}{|c|c|c|c|c|c|c|c|c|}
\hline Fonte de variação & gl & MTC $^{1}$ & & MTT & & MMT & Vigor & \\
\hline Genótipo (G) & 12 & 316,59 & $*$ & 427,06 & $*$ & 2775,14 & 4,05 & $*$ \\
\hline Ambiente (E) & 1 & 4108,88 & $*$ & 3150,84 & $*$ & 34537,10 & 0,15 & \\
\hline $\mathrm{G} \times \mathrm{E}$ & 12 & 193,73 & $*$ & 167,56 & $*$ & 602,55 & 1,77 & $*$ \\
\hline Resíduo & 72 & 13,80 & & 22,91 & & 59,84 & 0,28 & \\
\hline Média & - & 9,09 & & 17,04 & & 61,34 & 4,13 & \\
\hline $\mathrm{CV}(\%)$ & - & 16,69 & & 28,08 & & 12,60 & 12,85 & \\
\hline \multirow[t]{2}{*}{$\mathrm{CVg} / \mathrm{CV}$} & - & 1,65 & & 1,48 & & 2,38 & 1,29 & \\
\hline & & Ciclo & & COR & & $\mathrm{PE}$ & - & \\
\hline Genótipo (G) & 12 & 5,52 & $*$ & 14,21 & $*$ & 0,00092 & - & \\
\hline Ambiente (E) & 1 & 258,61 & $*$ & 30,15 & $*$ & 0,00261 & - & \\
\hline$G \times E$ & 12 & 2,90 & $*$ & 1,90 & $*$ & 0,00012 & - & \\
\hline Resíduo & 72 & 0,26 & & 0,30 & & 0,00009 & - & \\
\hline Média & - & 6,53 & & 5,88 & & 1,079 & - & \\
\hline $\mathrm{CVe}(\%)$ & - & 7,80 & & 9,31 & & 0,87 & - & \\
\hline $\mathrm{CVg} / \mathrm{CV}$ & - & 1,58 & & 2,40 & & 1,08 & - & \\
\hline
\end{tabular}

${ }^{1}$ MTC: massa de tubérculos comerciais $\left(\mathrm{t} \mathrm{ha}^{-1}\right)$; MTT: massa total de tubérculos $\left(\mathrm{t} \mathrm{ha}^{-1}\right)$; MMT: massa média de tubérculo (g tubérculo ${ }^{-1}$ ); Vigor: vigor de planta (1- alto a 9- baixo); Ciclo: ciclo de desenvolvimento vegetativo (1- tardio a 9- precoce); Cor: cor de fritura (1- escura a 9- clara); PE: peso específico. CVe: coeficiente de variação ambiental. $\mathrm{CVg} / \mathrm{CVe}$ : relação entre coeficiente de variação genotípico e ambiental. "Significativo a 5\% de probabilidade. 
Tabela 2. Médias de caracteres agronômicos, caracteres de qualidade de fritura, e incidência de requeima (Phytophthora infestans) para 11 clones canadenses e duas cultivares de batata, na safra de primavera de 2019, em Canoinhas, SC, e Pelotas, RS, Brasil.

\begin{tabular}{|c|c|c|c|c|c|c|c|c|}
\hline \multirow[b]{2}{*}{ Genótipo } & \multicolumn{2}{|l|}{ MTC $^{1}$} & \multicolumn{2}{|c|}{ MTT } & \multicolumn{2}{|l|}{ MMT } & \multicolumn{2}{|c|}{ Vigor } \\
\hline & Canoinhas & Pelotas & Canoinha & Pelotas & Canoinhas $\mathbf{P}$ & Pelotas & Canoinhas & Pelotas \\
\hline BGB 476 & 36,43 a $^{2}$ & $3,50 \mathrm{~b} \mathrm{~B}$ & 42,86 a $\mathrm{A}$ & $12,10 \mathrm{~b} \mathrm{~B}$ & B 121,12 a A & A $52,55 \mathrm{~b} \mathrm{~B}$ & $2,00 \mathrm{c} \mathrm{B}$ & 4,50 a $\mathrm{A}$ \\
\hline Markies & 33,10 a A & 5,39 a $\mathrm{B}$ & 39,53 a A & 21,18 a B & $3 \quad 123,15$ a $A$ & A 62,38 a B & $2,75 \mathrm{c} \mathrm{A}$ & $3,25 \mathrm{~b} \mathrm{~A}$ \\
\hline Asterix & $26,67 \mathrm{~b} A$ & $4,45 \mathrm{~b} \mathrm{~B}$ & $33,09 \mathrm{~b} \mathrm{~A}$ & 15,74 a B & $3 \quad 102,95 \mathrm{~b} \mathrm{~A}$ & A 57,70 a B & $2,50 \mathrm{c} \mathrm{B}$ & $3,50 \mathrm{~b} \mathrm{~A}$ \\
\hline BGB 479 & $23,57 \mathrm{~b} \mathrm{~A}$ & 6,70 a $B$ & $30,24 \mathrm{~b} \mathrm{~A}$ & $13,28 \mathrm{~b} \mathrm{~B}$ & B $108,19 b$ A & A 49,57 b B & 4,75 a A & 4,75 a $\mathrm{A}$ \\
\hline BGB 485 & $16,43 \mathrm{c} \mathrm{a}$ & $0,15 \mathrm{cb}$ & $29,76 \mathrm{~b}$ a & $9,88 \mathrm{~b} \mathrm{~b}$ & $70,49 \mathrm{~d} a$ & $32,38 \mathrm{c} \mathrm{b}$ & $3,50 \mathrm{~b}$ a & $4,25 \mathrm{a} a$ \\
\hline BGB 484 & $13,33 \mathrm{c} \mathrm{a}$ & $3,78 \mathrm{bb}$ & $20,72 \mathrm{c} \mathrm{a}$ & $12,05 \mathrm{~b} \mathrm{~b}$ & b $85,45 \mathrm{c} \mathrm{a}$ & $45,19 \mathrm{~b} \mathrm{~b}$ & $3,50 \mathrm{~b}$ a & $3,50 \mathrm{~b} \mathrm{a}$ \\
\hline BGB 482 & $10,72 \mathrm{c} \mathrm{a}$ & $1,81 \mathrm{cb}$ & $15,48 \mathrm{c} \mathrm{a}$ & $12,15 \mathrm{~b} \mathrm{a}$ & a $76,66 \mathrm{c} \mathrm{a}$ & $43,09 \mathrm{c} \mathrm{b}$ & 5,00 a a & $4,00 \mathrm{ab}$ \\
\hline BGB 475 & $10,48 \mathrm{c} \mathrm{a}$ & $4,96 \mathrm{ab}$ & $17,14 \mathrm{c} \mathrm{a}$ & $12,88 \mathrm{~b} \mathrm{a}$ & a $\quad 69,19 \mathrm{~d}$ a & $50,23 \mathrm{~b} \mathrm{~b}$ & 5,00 a a & $4,00 \mathrm{ab}$ \\
\hline BGB 477 & $8,81 \mathrm{~d} \mathrm{a}$ & $2,16 \mathrm{cb}$ & $13,81 \mathrm{~d} \mathrm{a}$ & $8,34 \mathrm{~b} \mathrm{a}$ & $66,20 \mathrm{~d} a$ & $39,23 \mathrm{c} \mathrm{b}$ & 5,00 a a & 4,75 a a \\
\hline BGB 480 & $7,62 \mathrm{da}$ & $1,67 \mathrm{c} \mathrm{b}$ & $21,43 \mathrm{c} \mathrm{a}$ & $9,61 \mathrm{~b} \mathrm{~b}$ & 52,73 e a & $31,75 \mathrm{c} \mathrm{b}$ & 4,75 a a & 4,25 a a \\
\hline BGB 481 & $5,00 \mathrm{~d} \mathrm{a}$ & $0,56 \mathrm{c} \mathrm{a}$ & $11,19 \mathrm{~d}$ a & $9,86 \mathrm{~b}$ a & 55,47 e a & $36,89 \mathrm{c} \mathrm{b}$ & 4,75 a a & $4,50 \mathrm{a} a$ \\
\hline BGB 483 & $4,05 \mathrm{~d} \mathrm{a}$ & $1,46 \mathrm{c} \mathrm{a}$ & $6,91 \mathrm{~d} \mathrm{a}$ & $8,06 \mathrm{~b}$ a & $62,07 \mathrm{~d} \mathrm{a}$ & $39,79 \mathrm{c} \mathrm{b}$ & 4,75 a a & 4,25 a a \\
\hline BGB 478 & $3,81 \mathrm{~d} \mathrm{a}$ & $0,00 \mathrm{c} \mathrm{a}$ & $10,95 \mathrm{~d} a$ & $4,87 \mathrm{~b} a$ & $40,78 \mathrm{f} \mathrm{a}$ & $19,90 \mathrm{~d} \mathrm{~b}$ & 5,00 a a & 4,75 a a \\
\hline Média & 15,38 & 2,81 & 22,55 & 11,54 & 79,57 & 43,13 & 4,10 & 4,17 \\
\hline $\mathrm{CV}(\%)$ & 33,01 & 48,07 & 27,51 & 23,52 & 12,35 & 11,13 & 11,34 & 14,16 \\
\hline $\mathrm{CVg} / \mathrm{CV}$ & 2,13 & 1,50 & 1,79 & 1,39 & 2,66 & 2,37 & 2,31 & 0,69 \\
\hline
\end{tabular}

${ }^{1}$ MTC: massa de tubérculos comerciais $\left(\mathrm{t} \mathrm{ha}^{-1}\right)$; MTT: massa total de tubérculos $\left(\mathrm{t} \mathrm{ha}^{-1}\right)$; MMT: massa média de tubérculo (g tubérculo $\left.{ }^{-1}\right)$; Vigor: vigor de planta (1- alto a 9- baixo); Ciclo: ciclo de desenvolvimento vegetativo (1- tardio a 9- precoce); Cor: cor de fritura (1- escura a 9- clara); PE: peso específico; Requeima (\%): porcentagem de plantas com requeima. ${ }^{2}$ Médias seguidas pela mesma letra minúscula na coluna e maiúscula na linha, pertencem ao mesmo grupo pelo teste de Scott-Knott, a 5\% de probabilidade do erro. 


\section{Continue a Tabela 2}

\begin{tabular}{|c|c|c|c|c|c|c|c|}
\hline \multirow[b]{2}{*}{ Genótipo } & \multicolumn{2}{|c|}{ Ciclo } & \multicolumn{2}{|c|}{ Cor } & \multicolumn{2}{|c|}{ PE } & \multirow{2}{*}{$\frac{\text { Requeima }(\%)}{\text { Pelotas }}$} \\
\hline & Canoinhas & S Pelotas & Canoinhas & Pelotas & Canoinhas & Pelotas & \\
\hline BGB 480 & 9,00 a a & $5,50 \mathrm{ab}$ & $6,00 \mathrm{c} \mathrm{a}$ & $3,50 \mathrm{cb}$ & $1,065 \mathrm{~b} \mathrm{a}$ & $1,060 \mathrm{~b} a$ & $26,25 \mathrm{a}$ \\
\hline BGB 481 & 9,00 a a & $5,50 \mathrm{ab}$ & $6,00 \mathrm{c} \mathrm{a}$ & $4,00 \mathrm{c} \mathrm{b}$ & $1,068 \mathrm{~b} \mathrm{~b}$ & $1,087 \mathrm{a} a$ & $28,75 \mathrm{a}$ \\
\hline BGB 478 & 9,00 a a & $5,00 \mathrm{ab}$ & $5,50 \mathrm{c} \mathrm{a}$ & $5,50 \mathrm{~b}$ a & 1,092 a a & $1,092 \mathrm{a} a$ & 7,50 a \\
\hline BGB 483 & 9,00 a a & $5,00 \mathrm{ab}$ & $5,50 \mathrm{c} \mathrm{a}$ & $5,00 \mathrm{~b}$ a & $1,075 \mathrm{a} \mathrm{a}$ & $1,084 \mathrm{a} a$ & $52,50 \mathrm{a}$ \\
\hline BGB 482 & 9,00 a a & $5,25 \mathrm{ab}$ & $7,50 \mathrm{~b} a$ & $6,00 \mathrm{~b} \mathrm{~b}$ & $1,067 \mathrm{~b} \mathrm{~b}$ & $1,084 \mathrm{a} \mathrm{a}$ & $53,75 \mathrm{a}$ \\
\hline BGB 475 & 8,75 a a & $5,00 \mathrm{ab}$ & $4,50 \mathrm{~d} b$ & $5,50 \mathrm{~b} a$ & 1,076 a a & $1,087 \mathrm{a} a$ & $22,50 \mathrm{a}$ \\
\hline BGB 479 & 8,50 a a & $5,00 \mathrm{ab}$ & 3,00 e a & $2,75 \mathrm{~d} a$ & $1,055 \mathrm{~b} \mathrm{a}$ & $1,058 \mathrm{~b} \mathrm{a}$ & $18,75 \mathrm{a}$ \\
\hline BGB 484 & 8,50 a a & $5,00 \mathrm{ab}$ & $7,00 \mathrm{~b} a$ & $5,75 \mathrm{~b} \mathrm{~b}$ & $1,081 \mathrm{a} \mathrm{b}$ & $1,095 \mathrm{a} a$ & $50,00 \mathrm{a}$ \\
\hline BGB 477 & 8,25 a a & $5,00 \mathrm{ab}$ & 8,50 a a & $7,25 \mathrm{ab}$ & $1,082 \mathrm{a} \mathrm{b}$ & 1,103 a a & $20,00 \mathrm{a}$ \\
\hline BGB 485 & 8,25 a a & $4,75 \mathrm{~b} \mathrm{~b}$ & $7,50 \mathrm{~b}$ a & 7,00 a a & $1,078 \mathrm{a} \mathrm{a}$ & $1,087 \mathrm{a} a$ & $31,25 \mathrm{a}$ \\
\hline BGB 476 & $7,50 \mathrm{~b} a$ & $4,25 \mathrm{~b} \mathrm{~b}$ & $7,50 \mathrm{~b} a$ & $6,00 \mathrm{~b} \mathrm{~b}$ & $1,081 \mathrm{a} \mathrm{a}$ & $1,094 \mathrm{a} \mathrm{a}$ & $11,25 \mathrm{a}$ \\
\hline Asterix & $6,75 \mathrm{c} \mathrm{a}$ & $4,75 \mathrm{~b} \mathrm{~b}$ & $7,00 \mathrm{~b}$ a & $5,25 \mathrm{~b} \mathrm{~b}$ & $1,080 \mathrm{ab}$ & $1,093 \mathrm{a} a$ & $32,50 \mathrm{a}$ \\
\hline Markies & $4,00 \mathrm{~d}$ a & $4,50 \mathrm{~b} a$ & 8,00 a a & $6,00 \mathrm{~b} \mathrm{~b}$ & $1,072 \mathrm{~b} \mathrm{a}$ & $1,078 \mathrm{a} a$ & $13,75 \mathrm{a}$ \\
\hline Média & 8,12 & 4,96 & 6,42 & 5,35 & 1,075 & 1,085 & 28,37 \\
\hline $\mathrm{CV}(\%)$ & 7,45 & 7,96 & 10,02 & 8,06 & 0,40 & 0,43 & 80,98 \\
\hline $\mathrm{CVg} / \mathrm{CV}$ & 2,28 & 0,74 & 2,34 & 2,95 & 7,12 & 0,82 & 0,45 \\
\hline
\end{tabular}

${ }^{1}$ MTC: massa de tubérculos comerciais $\left(\mathrm{t} \mathrm{ha}^{-1}\right)$; MTT: massa total de tubérculos $\left(\mathrm{t} \mathrm{ha}^{-1}\right)$; MMT: massa média de tubérculo ( $\mathrm{g}$ tubérculo $\left.{ }^{-1}\right)$; Vigor: vigor de planta (1- alto a 9- baixo); Ciclo: ciclo de desenvolvimento vegetativo (1- tardio a 9- precoce); Cor: cor de fritura (1- escura a 9- clara); PE: peso específico; Requeima (\%): porcentagem de plantas com requeima. ${ }^{2}$ Médias seguidas pela mesma letra minúscula na coluna e maiúscula na linha, pertencem ao mesmo grupo pelo teste de Scott-Knott, a 5\% de probabilidade do erro. 
Em relação à porcentagem de plantas com requeima, o valor desta relação $\mathrm{CVg} / \mathrm{CV}$ foi inferior à $50 \%$, indicando forte influência ambiental e baixa precisão experimental para este caráter, o que ajuda a explicar a ausência de diferenças significativas entre os genótipos.

Para os demais caracteres, a presença de valores de $\mathrm{CV}$ mais baixos ou da relação $\mathrm{CVg} / \mathrm{CV}$ com valores superiores, indicam que é possível obter conclusões confiáveis neste estudo (Tabelas 1 e 2).

$\mathrm{O}$ rendimento de tubérculos foi maior em Canoinhas, o que pode ser atribuído em parte à incidência de requeima no experimento de Pelotas, que acometeu, em média, $28,37 \%$ das plantas.

Para entender melhor o efeito ocasionado pela doença em relação aos demais caracteres, foi feita uma análise de correlação simples dos caracteres agronômicos com a incidência de requeima, cujos coeficientes não foram significativos, exceto com vigor de plantas $\left(\mathrm{r}=0,40^{* *}\right)$, isto é, parcelas com maior percentagem de plantas acometidas pela doença tenderam a apresentar plantas de vigor menor, afetando, consequentemente, $\mathrm{o}$ rendimento de tubérculos. Trabalhos têm demonstrado associação entre vigor das plantas de batata com produção de tubérculos (Khayatnezhad et al., 2011; Fekadu et al., 2013; Pereira et al., 2017).

A média do rendimento comercial de tubérculos em Canoinhas foi 15,38 t.ha- ${ }^{-1}$, havendo uma grande variação de rendimento entre os genótipos, com valores superiores para o clone BGB 476 (36,43 t.ha $\left.^{-1}\right)$ e a testemunha Markies (33,10 t.ha th $\left.^{-1}\right)$, e inferiores para os clones BGB $478\left(3,81\right.$ t.ha $\left.{ }^{-1}\right)$, BGB $483(4,05$ t.ha-1), BGB $481\left(5,00\right.$ t.ha $\left.^{-1}\right)$, BGB 480 $\left(7,62\right.$ t.ha $\left.{ }^{-1}\right)$, BGB $477 \quad\left(8,81\right.$ t.ha $\left.{ }^{-1}\right)$ (Tabela 2). BGB 476 e Markies apresentaram também as maiores massa média de tubérculos e massa total de tubérculos.

Apesar do menor rendimento de tubérculos comerciais em Pelotas, BGB 475 (4,96 t.ha $\left.{ }^{-1}\right)$ e BGB 479 (6,70 t.ha $\left.{ }^{-1}\right)$ apresentaram os maiores rendimentos, inclusive superiores às cultivares testemunhas.

A maioria dos clones apresentaram plantas menos vigorosas (plantas de menor tamanho) e com ciclo vegetativo mais precoce do que as cultivares, indicando que este germoplasma apresenta potencial para programas de melhoramento que busquem estas características.

Quanto aos caracteres de qualidade de fritura, cor de fritura mais clara e maior peso específico combinados, e em ambos os experimentos, observa-se superioridade do clone BGB 477 em relação aos demais genótipos. Outros clones que se destacaram para qualidade de fritura em comparação com as testemunhas foram BGB 476, BGB $484 \mathrm{e}$ BGB 485.

Em avaliação de outro conjunto de clones de origem canadense, Silva et al. (2019) identificaram clones superiores para a qualidade de fritura e rendimento de tubérculos, mas, em média, com plantas mais vigorosas.

Genótipos de batata originados de regiões com fotoperíodo longo experimentam redução de ciclo de desenvolvimento vegetativo, antecipação da tuberização e maior competição por fotoassimilados pelos tubérculos (enchimento) em relação ao crescimento vegetativo quando plantados em regiões de dias mais curtos 
(Pinto et al., 2010; Silva et al., 2012). Esta hipótese foi confirmada neste estudo, na medida em que, em geral, as médias de ciclo vegetativo e as médias de vigor de planta dos clones canadenses foram maiores (mais precoces e menos vigorosas) do que as médias das cultivares testemunhas, as quais foram selecionadas em condições de fotoperíodo longo, na Europa.

Muito embora tenham sido identificados clones com elevada produtividade de tubérculos comerciais, nenhum dos clones superou a ambas as cultivares, nos dois experimentos. BGB 476, em Canoinhas, foi o clone mais produtivo e com tubérculos de maior massa média, mas não diferiu da cultivar testemunha Markies. Este clone apresentou plantas com baixo vigor (plantas pequenas), mas sem diferir das testemunhas, e ciclo vegetativo mais curto (mais precoce) do que ambas as testemunhas.

As correlações, levando em consideração todas as observações, não foram significativas entre rendimento comercial e ciclo vegetativo, mas foi significativa entre rendimento comercial e vigor de planta $(\mathrm{r}=-0,57 * *)$. Há uma tendência de relação direta de ciclo vegetativo e vigor de planta com rendimento de tubérculos, isto é, os genótipos de ciclo longo (>130 dias) serem mais produtivos que genótipos precoces (<100 dias), provavelmente pelo maior tempo para sintetizar e armazenar fotoassimilados nos tubérculos (Silva \& Pinto, 2005; Rodrigues et al., 2009; Silva et al., 2018). Por maior duração da área foliar, genótipos de ciclo mais longo apresentam maior produtividade (Silva et al., 2009; Rolando et al., 2015). Por outro lado, os produtores preferem cultivares mais precoces, por possibilitarem maior número de cultivos por ano na mesma área, com menor tempo de exposição das plantas a intempéries, menor risco de doenças e pragas, e menor demanda de irrigação (Dias et al., 2003; Rodrigues et al., 2009).

Conclui-se que no germoplasma canadense avaliado neste trabalho, há clones com características potenciais, como o rendimento de tubérculos, vigor de planta (tamanho), ciclo vegetativo e qualidade de fritura adequados ao cultivo no Sul do Brasil. BGB 476 é o clone mais destacado, com elevado rendimento de tubérculos e boa qualidade de fritura. E, tem ciclo vegetativo e vigor de plantas similares às principais cultivares utilizadas no país para processamento de palitos pré-fritos congelados.

\section{Conflitos de interesse}

Esta pesquisa não apresenta conflitos de interesse.

\section{Referências}

Cabezas-Serrano, A.B.; Amodio, M.L.; Cornacchia, R.; Rinaldi, R.; Colelli, G. (2009). Suitability of five different potato cultivars (Solanum tuberosum L.) to be processed as fresh-cut products. Postharvest Biology and Technology 53:138-144.

Cruz, C.D. (2013). Genes; a software package for analysis in experimental statistics and quantitative genetics. Acta Scientiarum. Agronomy 35:271-276.

Cruz, C.D.; Regazzi, A.J.; Carneiro, P.C.S. (2014). Modelos biométricos aplicados ao melhoramento genético. Terceira edição. Universidade de Viçosa, Viçosa, MG. 668 p.

Dias, G.S.; Silva, E.C.; Maciel, G.M. (2003). Competição de cultivares de 
batata na Região de Alfenas-MG. Horticultura Brasileira, Brasília 21:310.

Fekadu, A.; Petros, Y.; Zelleke, H. (2013). Genetic variability and association between agronomic characters in some potato (Solanum tuberosum L.) genotypes in SNNPRS, Ethiopia. International Journal of Biodiversity and Conservation 5:523-528.

Fernandes, A.M.; Sorrato, R.P.; Evangelista, R.M.; Nardim, I. (2010). Qualidade fisíco-química e de fritura de tubérculos de cultivares de batata na safra de inverno. Horticultura brasileira 28:299-304.

Freitas, S.T.; Bisognin, D.A.; Gómeza, C.S.; Sautter, C.K.; Costa, L.C.; Rampelotto, M.V. (2006). Qualidade para processamento de clones de batata cultivados durante a primavera e outono no Rio Grande do Sul. Ciência Rural 36:80-85.

Haase, N.U. (2008). Healthy aspects of potatoes as part of the human diet. Potato Research 51:239-258.

Khayatnezhad, M.R.; Shahriari, B.R.; Gholamin, R.G. (2011). Correlation and path analysis between yield and yield components in potato (Solanum tuberosum L.). Middle-East Journal of Scientific Research 7:17-21.

Kumar, P.; Pandey, S.; Singh, B.; Singh, S.; Kumar, D. (2007). Influence of source and time of potassium application on potato growth, yield, economics and crisp quality. Potato Research 50:1-13.

Lyra, D.H.; Ribeiro, G.H.M.R.; Figueiredo, I.C.R.; Guedes, M.L.; Carneiro, O.L.G.; Pinto, C.A.B.P.; Pereira, A. (2015) Início da tuberização, duração do ciclo vegetativo e tolerância ao calor em genótipos de batata. Pesquisa Agropecuária Brasileira 50:582-592.
Moreira, C.M.; Pinto, C.A.B.P.; Ribeiro, G.H.M.R.; Carneiro, O.L.G.; Guedes M.L. (2015). Clones de batata tolerantes ao calor para diferentes segmentos de mercado. Amazonian Journal of Agricultural and Environmental Sciences 58:138-145.

Pereira, A. da S. (org.). (2010). Produção de batata no Rio Grande do Sul. Embrapa Clima Temperado, Pelotas, RS, Brasil. 95 p.

Pereira, A.S.; Silva, G.O. (2019). Batata: evolução na oferta de cultivares brasileiras e na produção de tubérculossemente. Seed News 23:36-39.

Pereira, A. da S.; Silva, G.O.; Carvalho, A.D.F.; Ponijaleki, R.S. (2017). Performance of advanced potato clones: plant vigor, tuber yield and specific gravity. Horticultura Brasileira 35:440444.

Pinto, C.A.B.P.; Teixeira, A.L.; Neder, D.G.; Araújo, R.R.; Soares, A.R.O.; Ribeiro, G.H.M.R.; Lepre, A.L. (2010) Potencial de clones elite de batata como novas cultivares para Minas Gerais. Horticultura Brasileira 28:399-405.

Rodrigues, G.B.; Pinto, C.A.B.; Benites, F.R.G.; Melo, D.S. (2009). Seleção para duração do ciclo vegetativo em batata e relação com a produtividade de tubérculos. Horticultura Brasileira 27:280-285.

Rolando, J.L.; Ramírez, D.A.; Yactayo, W.; Monneveux, P.; Quiroz, R. (2015). Leaf greenness as a drought tolerance related trait in potato (Solanum tuberosum L.). Environmental and Experimental Botany 110:27-35.

Schippers, P.A. (1976). The relationship between specific gravity and percentage of dry matter in potato tubers. American Potato Journal 53:111-122. 
Silva, L.A.S.; Pinto, C.A.B.P. (2005). Duration of the growth cycle and the yield potential of potato genotypes. Crop Breeding and Applied Biotechnology 5:20-28.

Silva, G.O.; Pereira, A. da S.; Souza, V.Q.; Carvalho, F.I.F.; Neto, R.F. (2006). Early generation selection for tuber appearance affects potato yield components. Crop Breeding and Applied Biotechnology 6:73-78.

Silva, G.O.; Castro, C.M.; Terres, L.R.; Rohr, A.; Suinaga, F.A.; Pereira, A. da S. (2012). Desempenho agronômico de clones elite de batata. Horticultura Brasileira 30:557-560.

Silva, G.O.; Pereira, A. da S.; Azevedo, F.Q.; Carvalho, A.D.F.; Pinheiro, J.B. (2018). Selection of potato clones for tuber yield, vine maturity and frying quality. Horticultura Brasileira 36:276281.

Silva, G.O.; Pereira, A. da S.; Azevedo, F.Q.; Carvalho, A.D.F.; Pinheiro, J.B. (2019). Selection of Canadian potato clones for agronomic and frying quality traits. Horticultura Brasileira 37:423-428.

Silva, F.L.; Pinto, C.A.B.P.; Alves, J.D.; Benites, F.R.G.; Andrade, C.M.;
Rodrigues, G.B.; Lepre, A.L.; Bhering, L.L. (2009). Caracterização morfofisiológica de clones precoces e tardios de batata visando à adaptação a condições tropicais. Bragantia 68:295302.

Smith, O. (1975). Potato chips. pp. 305402. In: Talburt, W.F.; Smith, O. (eds.). Potato processing. Terceira edição. AVI, Westport. 705 p.

Souza, Z.S; Bisognin, D.A; Junior, G.R.M; Gnocato, F.S. (2011). Seleção de clones de batata para processamento industrial em condições de clima subtropical e temperado. Pesquisa Agropecuária Brasileira 46:1503-1512.

Stark, J.C.; Love, S.L. (2003). Tuber quality. pp. 329-343. In: Stark, J.C.; Love, S.L. (eds.). Potato production systems. University of Idaho, Aberdeen, ID, Estados Unidos. 420 p.

Tiemens-Hulscher, M.; Delleman, J.; Eising, J.; Lammmerts Van Bueren, E. (2003). Potato breeding: A practical manual for the potato chain. Wageningen University, Wageningen, The Netherlands. 172 p. 